\title{
EFFICIENT REORIENTATION OF A DEFORMABLE BODY IN SPACE: A FREE-FREE BEAM EXAMPLE
}

\author{
llya V. Kolmanovsky and N. Harris Mc ('lamroch* \\ Dept. of Aerospare Engineering \\ Iniversity of Mirhigan. Ann Arbor. MI $48109-2140$
}

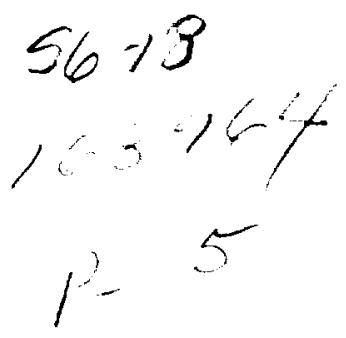

\begin{abstract}
It is demonstrated that the planar reorientation of It is demonstrated that the planar reorientation of complished by periodically changing the shape of the beam using internal actuators. A control scheme is proposed in which electromechanical actuators excite the flexible motion of the beam so that it rotates in the desired manner with respect to a fixed inertial reference. The results can be viewed as an extension of previous work to a distributed parameter case
\end{abstract}

\section{Introduction}

Following [8], we introduce the concept of a deformable body, for which distances between the points of the body can change during the motion. Examples of deformable bodies include both lumped and distributed parameter systems such as multilink rigid body interconnections and structures with distributed flexibility. The orientation of a deformable body with respect to a fixed inertial reference can be specified by a choice of body frame. In general. there are many ways to choose a body frame. For example. in the case of planar motion a body frame can be identified with any two distinct points in the body. The shape of a deformable body can be specified in terms of the position of the body relative to the body frame Thus, an arbitrary motion of a deformable body can be separated into rigid body motion and shape change.

Assume that both linear and angular momenta about the center of mass of the body are conserved and equal to zero. These conditions hold if the body is in a circular orbit around the Earth or is in a free fall. As a consequence of angular momentum conservation, shape change and the rigid body motion are coupled. This coupling is inherently nonlinear. In couple nne mav he interested in inducing a ro-
In this paper we extend the aforementioned reorientation strategies to the case of flexible bodies. In tation strategies interested in a planar reorientation of a free-free beam in space using only electromechanical actuators. These electromechanical actuators e.g. piezoelectric or shape memory actuators do not change the angular momentum of the freedo not change the be used to change the shape of the beam in a periodic way. Assuming that the angular beam in a periodic beam is always zero, oscillations in the shape of the beam can cause a rotation of the beam with respect to a fixed inertial reference. The rotation of the beam over one period depends only on the shape of the beam over one period and does not depend on the length of the period; hence this phenomenon is referred to as a geometric phase change.

The extension of existing strategies to the free-free beam case is not straightforward for several reasons. Classical models of uniform free-free tlexible beams in zero gravity space result in complete decoupling of rigid body motion and flexible motion. Higher order nonlinear coupling between rigid body motion and flexible motion is captured in geometrically exact beam theories [9]. The resulting models, however, are complicated. The free-free beam is an infinite dimensional superarticulated system. Thus, an arbitrary shape change cannot be produced by a finite number of actuators. In addition, the body frame of the beam needs to be chosen so that the shape change is independent of the rigid body motion. Such a choice of pendent of the rigid for lumped parameter systems since variables specifying orientation are ignorable

In this paper, we first address basic modeling issues. The dynamics which determine the shape of the free-free beam are assumed to be characterizel by the Euler-Bernoulli equation, including material damping, with appropriate boundary conditions. The higher order coupling between the rigid body motion and the flexible motion is captured using the angular momentum expression which includes rotatory iner-

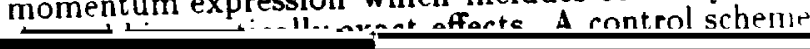


frame with its origin fixed at the origin of the inertial frame such that the vectors $(i, \bar{k})$ lie in the plane $\left(\bar{e}_{1}, \bar{r}_{3}\right)$ and $\bar{j}=\bar{e}_{2}$. The straight line passing through the origin in the direction of vector $k$ is called the reference line. Let the beam initially be at rest in a straight line configuration aligned with the reference line. Then, the location of each point on the line of mass centroids of the beam can be described in terms of the parameter $s \in[-L, L]$. This parameter s ran be viewed as a label for each of the crossections. We assume that as the beam deforms the shape and the area of the crossections remain invariant. Following other researchers $[1,6,9]$ we introduce three functions $u(s, t), y(s, t):[-L . L] \times R \rightarrow R$ and $t(s, t)$ $[-L, L] \times \Re \rightarrow T^{l}$ such that $(u(s . l)+s . y(s . l))$ define the coordinates of the line of centroids in the deformed configuration with respect to the moving frame $(\bar{i}, \bar{k})$ at time $t$. The angle $l:(s, t)$ between the normal to the crossection at $s$ and $r_{3}$ specifies the orientation of the crossection. The normal to the crossection at $s$ is denoted by $\bar{t}_{3}$. We define the material basis $\left(\bar{t}_{1}, \bar{t}_{2}, \bar{t}_{3}\right)$ to be or thonormal so that $\bar{t}_{1}$ lies in the plane $\left(\bar{e}_{1}, \bar{e}_{3}\right)$. The crossection itself can be associated with the set of points $\left(\xi_{1}, \xi_{2}\right)$ in a compact set $A \subset \Re^{2}$ such that $\xi_{1} \bar{t}_{1}+\xi_{2} \bar{t}_{2}+(u(s, t)+s) \bar{k}+(y(s, t)) \bar{i}$ gives the location of any point on the beam as $\xi_{1}$ and $\xi_{2}$ vary through $A$ and $s$ varies from $-L$ to $L$.

Since the origin of the inertial frame is fixed at the center of mass of the beam we obtain

$$
\begin{aligned}
& \int_{-L}^{L} y(s, t) d s=0 \\
& \int_{-L}^{L} u(s, t) d s=0 .
\end{aligned}
$$

Let $\rho$ denote the constant mass density per unit volume of the beam. We assume that the beam has a symmetric crossection so that the first moment of inertia of the crossection about the line of centroids is

$$
\int_{1} \rho \xi_{1} d \xi_{1} d \xi_{2}=0
$$

The second moment of inertia of the crossection about the line of centroids. referred to as the rotatory inertia, is

$$
I_{2}=\int_{A} \rho \xi_{1}^{2} d \xi_{1} d \xi_{2}
$$

and assumed to be positive. The mass per unit length of the crossection is given by

$$
m_{0}=\int_{A} \rho d \xi_{1} d \xi_{2}
$$

We define the angle $\theta(t)$ between $\bar{e}_{3}$ and $\bar{k}$ so that $y(s, t)$ measured from the reference line satisfies the following orthogonality condition

$$
\int_{-L}^{L} s y(s, t) d s=0
$$

The existence of the angle $\theta(t)$ follows from the geometry indicated in Fig. 1. This definition provides a separation between the motion which determines the shape of the beam, given by $y(s, t),-L \leq s \leq L$, and the rotation of the beam as a whole, given by ant.

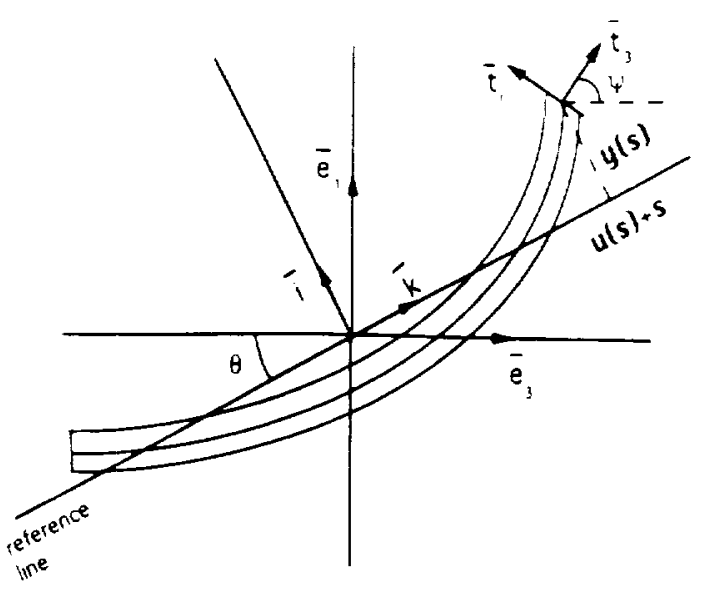

Fig l. Planar Beam Model

We next develop a kinematically exact expression for the angular momentum of the free-free beam. Let $F\left(s . \xi_{1}, \xi_{2}, \theta, t\right)$ be the vector from the origin of the inertial frame to a point $\left(s, \xi_{1}, \xi_{2}\right)$ on the beam at time $t$; then

$$
\begin{gathered}
\bar{\rho}=\left(s \sin \theta+y \cos \theta+\xi_{1} \cos \psi+u \sin \theta\right) \bar{e}_{1}+ \\
\left(\xi_{2}\right) \bar{e}_{2}+\left(s \cos \theta-\xi_{1} \sin \psi-y \sin \theta+u \cos \theta\right) \bar{e}_{3}
\end{gathered}
$$

where $\theta=\theta(t), y=y(s, t)$ and $\psi=\psi(s, t)$. The angular momentum about the origin of the inertial frame at time $t$ is zero so that

$$
\int_{-L}^{L} \int_{A} \rho \vec{\varphi} \times \frac{d \bar{\varphi}}{d t} d \xi_{1} d \xi_{2} d s=0
$$

Substituting equation (7) into equation (8) and using equations (4) and (5) we can express $\dot{\theta}$ in terms if $y . u$ and $\alpha$ as

$$
\dot{\theta}=\frac{\int_{-L}^{L}\left\{m_{0} s \frac{\partial y}{\partial t}+l_{2} \dot{\alpha}+m_{0}\left(\frac{\partial y}{\partial t} u-\frac{\partial u}{\partial t} y\right)\right\} d s}{\int_{-L}^{L}\left\{-m_{0} s^{2}-m_{0} y^{2}-I_{2}\right\} d s}
$$

where $\alpha=\psi-\theta$ is the angle between the normal $t_{3}$ to the crossection at $s$ and the reference line.

Assume that the beam is unshearable and inextens:ble and that the deformations are small. This implies using equation (2), that

$$
u(s, t)=0 .
$$

and that

$$
\alpha \approx y_{s}
$$

We use the Euler-Bernoulli beam model to chara: terize the shape of the beam [3]. Thus $y(s, t)$ satistic the Euler-Bernoulli equation of the form

$$
m_{0} y_{t t}+\gamma y_{t s s s s}+E I y_{s s s}=-\sum_{j=1}^{m} v_{j}(t) \delta^{\prime}\left(s-s_{j}\right)
$$


non-straight line reference configuration. It follows from expression (18) that in order to rotate the beam in the opposite direction it is sufficient to reverse the signs of $v_{j}$ and $v_{j}^{0}$.

We are now in a position to formulate a specific control strategy to accomplish the desired asymptotic manellver. Starting at rest with $A\left(t_{n}\right)=A_{n}$ application of control law (19) results in a nonzern geometric phase change over one period. By repetiriun of cyrles of motion as many times as necessary the brani can be caused to rotate closer and closer to $H_{t}$. Is $A(t)$ approach $\theta_{d}$ we can reduce the amplitude of the iscillations to zero in a way so that $\theta(t) \rightarrow A_{1}$ as $t-x$

The proposed control law is of the firm

$$
v_{j}(t)=\varepsilon_{k}\left[\bar{v}_{j}^{0}+\bar{i}_{j} \cos (\omega t)\right] . j=1 \ldots \ldots . \quad(2 ;)
$$

where $\frac{2(k-1) \pi p}{\omega} \leq t-t_{0}<\frac{2 k \pi p}{\omega}, k=1.2 \ldots$ that is, the control excitation is an amplitude motulated function, where $\bar{v}_{j}^{0}, \bar{v}_{j}^{w}$ are constants and $\Sigma_{k}$ denotes the scalar amplitude modulation sequence that defines the control excitation on the $k$-th cycle. Each cycle is exactly $p$ periods. The constants $\alpha, \bar{v}_{j}^{0}, \bar{v}_{j}$ can be chosen nearly arbitrary, although one approach is to choose $\bar{v}_{j}^{0}, \bar{v}_{j}^{\omega}$ to maximize geometric phase expression (22) where $a_{i}, l_{i}, \phi_{i}, i=1, \ldots$ are related to $i, j$, $\bar{v}_{j}, j=1, \ldots, m$ according to expressions $(20)$ and $(1 i)$, and $\bar{v}_{j}^{0}, \bar{v}_{j}^{\infty}$ are constrained in norm. In terms of $\bar{v}_{j}^{0}, \vec{v}_{j}^{w}, j=1, \ldots, m$ this is a constrained mathematical programming problem which is linear in $\tilde{v}_{j}^{0}$ (for fixed $\bar{v}_{j}^{\omega}$ ) and quadratic in $\bar{v}_{j}^{\omega}$ (for fixed $i_{j}^{0}$ ). We will subsequently denote the maximum value of this constrained optimization problem as $\lrcorner \theta^{*}$

The modulation sequence $\Sigma_{k+1}$ is defined in terms of an average of $\theta(t)$. over the $k$-th cycle. that is

$$
\theta_{k}^{\text {aze }}=\frac{1}{2}(\max \theta(t)+\min \theta(t))
$$

where the maximum and minimum are over $\frac{2 \mid k-1, T p}{4} \leq 1-t_{0} \leq \frac{2 k \pi p}{u}$. We also introduce two auxilary variables $\theta_{0}^{a \cdot}=\theta_{0}$ and $\varepsilon_{0}=\operatorname{sign}\left(\frac{\theta_{x}-\theta_{0}}{\Delta \theta^{\circ}}\right)$ We express $\varepsilon_{k}$ in terms of $\theta_{k-1}^{a v e}$ and $\varepsilon_{k-1}$ as indicated below:

(A1) compute

$$
r_{k}=\left(\frac{\theta_{d}-\theta_{k-1}^{\text {ave }}}{\Delta \theta^{*}}\right)^{\frac{1}{3}}
$$

(A2) In case $\left|r_{k}\right| \geq\left|\varepsilon_{k-1}\right|$, if $r_{k}$ and $\varepsilon_{k-1}$ have the same signs then $\varepsilon_{k}=\left|\varepsilon_{k}-i\right| j \operatorname{sign}\left(r_{k}\right)$; if $r_{k}$ and $\varepsilon_{k-1}$ have opposite signs then $\varepsilon_{k}=$ $\gamma_{1}\left|\varepsilon_{k-1}\right| \operatorname{sign}\left(r_{k}\right)$. where $0<\gamma_{1}<1$.

(A3) If $0<\left|r_{k}\right|<\left|\Sigma_{k-1}\right|$ then $\varepsilon_{k}=\gamma_{2} r_{k}$, where $0<\gamma_{2}<1$.

(A4) If $r_{k}=0$ then $\varepsilon_{k}=\Sigma_{k-1}$
Proposition 4.2 If the proposed control law is of the form $(2: 3)$ where ${ }_{x}$ is selected accurding $i$, steps (A1)-(A4), then

$$
\lim _{k \rightarrow x} \theta_{k}^{\text {ave }}=\theta_{t} \cdot \lim _{k \rightarrow \infty} \sum_{k}=0 .
$$

Sketch of the Proof. The sequence $\hat{k}_{k}$ is nun increasing and bounded on $[0,1]$. Therefore. there exists $b \in[0,1]$ such that $b=\inf _{k}\left|\varepsilon_{k}\right|$. It ratu l, shown that by construction of the sequence by minet be zero.

Since $|=k|-0$ then $\varphi_{i}(t)-0$ and $\dot{q}_{i}-0$ as $t-x$. By continuity $\theta(t)-\theta^{\text {con }}$ for some constant $\theta^{\circ}$ it. $1-x$. It can be shown that $\theta^{c o n}=\theta_{d}$.

Finally, it follows from equations (24) and (20) that

$$
\lim _{t \rightarrow \infty} \theta(t)=\theta_{d} \cdot \lim _{t \rightarrow \infty}\left(\begin{array}{l}
y(s, t) \\
y_{t}(s, t)
\end{array}\right)=0,-L \leq s \leq L
$$

The controller which we have constructed has two functions. Its main function is to excite the oscillations of the beam in such a way that the beam rotates in the desired sense. Subsequently, the controller serves to suppress the vibrations previously excited so that the free-free beam comes to rest with a desired orientation. Note that control law (23) is a non-smooth feedback control law [2].

\section{Numerical Example}

Consider a beam with half-length $L=1[\mathrm{~m}]$. density per unit volume $\rho=1400\left[\mathrm{~kg} / \mathrm{m}^{3}\right]$ and squar crossection with the side size $R=0.1[\mathrm{~m}]$. Young : modulus of the beam is $E=3.0 \times 10^{6}\left[\mathrm{~N} / \mathrm{m}^{2}\right]$ and the Kelvin- Voigt damping coefficient is $\gamma=0.2$. T $w$ actuators are installed near both ends of the bean at $s_{1}=-0.9[\mathrm{~m}]$ and $s_{2}=0.9[\mathrm{~m}]$. The maxmm wrine pach of the actuators can produce is mplial $100[\mathrm{Ym}]$. The excitation frequency $\omega=13[\mathrm{H}=\mathrm{i}, \ldots$ lected to lie between the first $10.6[\mathrm{~Hz}]$ and the secund $29[\mathrm{~Hz}]$ resonant frequencies of the beam; $\bar{v}_{j}^{0}$ and $j=1.2$ are chosen using expression (22) to maximiz the geometric phase change over one period. For thin example we choose $p=5$ and $\gamma_{1}=\gamma_{2}=0.9$. $\quad\left[L_{1}\right.$. first four elastic modes of the beam are used in ...1r simulation.

We want to rotate the beam from $\theta_{0}=0.1[\mathrm{rad}]$ al $t=0[\mathrm{sec}]$ to $\theta_{d}=0[\mathrm{rad}]$. The dependence of th. angle $\theta(t)[\mathrm{rad}]$ on time $t[\mathrm{sec}]$ is shown for a part t the maneuver in Fig. 2. In this case the geometr. phase change over one period in steady-state 1,5 dicted by expression (22) is equal to $-2.7465 \times 10^{- \text {- }}$ [ $\mathrm{rad}]$ whereas its actual simulation value is equal $\mathrm{t}$. $-3.0411 \times 10^{-4}[\mathrm{rad}]$. The dependence of the mu. ulation parameter $\varepsilon$ on time is shown in Figur 


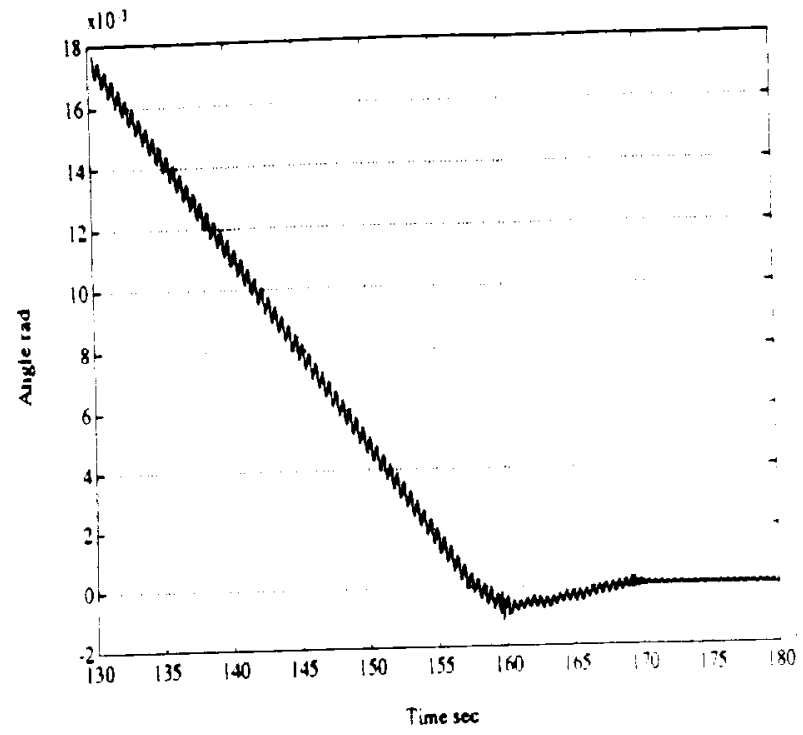

Fig 2. Asymptotic Reorientation Maneuver

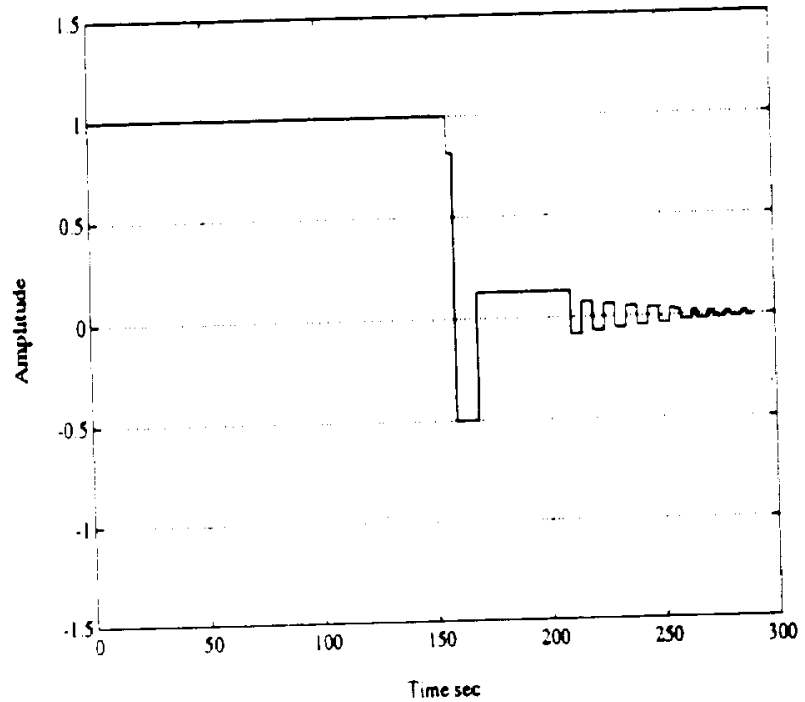

Fig 3. Amplitude Modulation Sequence

\section{Conclusion}

In this paper the angular momentum expression for a In shown how the general motion of the beam can be separated into rigid and elastic motions. The change of shape f the heam is described by the Euler-Bernoulli equa- beam by internal actuators are derived. Finally, a control strategy for a planar asymptotic reorientation maneuver is developed.

A general treatment of the interplay between deformations and rotations of deformable bodies is given by Shapere and Wilczek [ 8$]$. Reyhanoglu and Mc( lamroch $[\bar{l}]$ have developed a framework for reorientation of multibody systems in space. In this paper. we have used the framework developed by Shapere and Wilczek for the specific problem of reorientation of a free-free beam in space; our results represent. in a certain sense, the limiting case of the multibody re. sults obtained by Reyhanoglu and McClamroch when the number of bodies increases without limit.

Although our study in this paper has been concerned with the ideal case of reorientation of a free-free beam in space. we note that the same ideas are applicable to reorientation of a wide class of deformable space structures. using only actuators embedded into the structure. In this sense, smart structures technology can be used to accomplish a variety of efficient reorientation maneuvers for space structures.

\section{References}

[1] J. Baillieul and M. Levi, "Constrained relative motions in rotational mechanics," Arch. Rational Mech. Anal. Vol. 115, pp. 101-135, 1991.

[2] A. Bloch. M. Reyhanoglu and N. H. McClamroch. "Control and stabilization of nonholonomic dynamic systems," IEEE Transactions on Automatic Control, Vol. 37, No. 11, pp. 1746-17.7. 1990.

[3] Y. Ci. Fung, Foundations of Solid Mechanics, Prentice-Hall, 1965.

[4] P. S. Krishnaprasad, "Geometric phases, and optimal reconfiguration for multibody systems." Proc of the American Control Conference. Vol. 3, pp. 2440-2444, 1990.

(5) R. Murray and S. Sastry, "Steering nonholonomic systems using sinusoids," Proc. of the $29-1 \mathrm{~h}$ Conference on Decision and Control, Vol. 4. pp. $2097-2101,1990$.

[6] E. Reissner. "On a one-dimensional tinite strain beam theory: the plane problem." J. Appl. Wath. Phys., Vol. 23, pp. 795-804, 1972

[7] M. Reyhanoglu and N. H. McClamroch, "Planar reorientation maneuvers of space multibody systems using internal controls," $J$. Guidance. systems using internal Dynam, Vol. 15, No.6, pp. 1475$1480,199^{\circ}$.

[8] A. Shapere and F. Wilczek, "Gauge kinematics of deformable bodies," Geometric Phases in Physics, World Scientific, Singapore, pp. $449-$ $460,1989$.

[9] J. C. Simo and L. Vu-Quoc, "On the dynamics in space of rods undergoing large motions - A geo- 
I 